\title{
Hypersomnia in children affected by migraine without aura: a questionnaire-based case-control study
}

This article was published in the following Dove Press journal:

Neuropsychiatric Disease and Treatment

20 February 2013

Number of times this article has been viewed

\author{
Maria Esposito' \\ Michele Roccella ${ }^{2}$ \\ Lucia Parisi ${ }^{2}$ \\ Beatrice Gallai ${ }^{3}$ \\ Marco Carotenuto' \\ 'Center for Childhood Headache, \\ Unit of Child and Adolescent \\ Neuropsychiatry, Department \\ of Mental Health, Physical, and \\ Preventive Medicine, Second \\ University of Naples, Naples, Italy; \\ ${ }^{2}$ Child Neuropsychiatry, Department \\ of Psychology, University of Palermo, \\ Palermo, Italy; ${ }^{3}$ Unit of Child and \\ Adolescent Neuropsychiatry, \\ University of Perugia, Perugia, Italy
}

Correspondence: Marco Carotenuto Center for Childhood Headache, Clinic of Child and Adolescent Neuropsychiatry, Department of Mental Health, Physical and Preventive Medicine, Via Sergio Pansini 5 PAD XI, 80I3I Naples, Italy Tel +39815666988

Fax $+398 I 5666694$

Email marco.carotenuto@unina2.it
Background: The relationship between sleep and headache is meaningful and complex. Children affected by migraines tend to show many sleep disorders, such as insufficient sleep duration and excessive daytime somnolence. Therefore, the aim of this study is to assess the rate of reported sleep habits and self-reported sleepiness in a large pediatric sample of individuals affected by migraine without aura (MoA).

Methods: The study population consisted of 271 children aged between 6 and 13 years affected by MoA. The control group was composed of 305 typically developing children. To assess the sleep habits of all individuals (MoA and control), parents filled out the Sleep Disturbance Scale for Children, and to check the degree of subjective perceived daytime sleepiness, all subjects were administered the Pediatric Daytime Sleepiness Scale.

Results: The two study groups were matched for age $(P=0.124)$, sex distribution $(P=0.775)$, and body mass index z-score $(P=0.107)$. Parents of children affected by MoA reported a higher total score of sleep disorder symptoms $(P<0.001)$, disorders of initiating and maintaining $(P<0.001)$, and disorders of arousal $(P<0.001)$ than did parents of controls. No significant differences were found in disorders of excessive somnolence. Conversely, in the Pediatric Daytime Sleepiness Scale, migraine children had higher scores $(24.67 \pm 3.19$ vs $11.94 \pm 4.81 ; P<0.001)$ and a reduction in referred total sleep time mean duration (469.83 \pm 98.112 vs $527.94 \pm 83.02$; $P<0.001)$ than typically developing children.

Conclusion: Our study identified differences in sleep habits and found a high prevalence of daytime somnolence in children affected by MoA, suggesting the need for routine sleep screening in the pediatric management of children with migraines.

Keywords: excessive daytime sleepiness, drowsiness, sleep disorders, migraine without aura, children

\section{Introduction}

In 1873, Edward Liveing, in his work "On Megrim, Sick-Headache and Some Allied Disorders: A Contribution to the Pathology of Nerve-Storms," described the role of sleep as a treatment for migraine attacks, highlighting the link between the two processes. ${ }^{1}$ The interrelationship between sleep disorders and headache is well recognized, although still obscure and not yet fully clarified. In particular, migraine in children is not only a painful syndrome but is also often accompanied by many severe disabilities/ impairments, such as low emotional functioning, school absenteeism, impairment of academic performance and cognitive functioning, ${ }^{2,3}$ motor coordination, ${ }^{4}$ and sleep habits. ${ }^{5-8}$ Thus, in clinical practice, there are many alternative therapies that could be useful and promising for the treatment of headache symptoms in children, such as 
weight loss, ${ }^{9}$ treatment with nutraceuticals, ${ }^{10,11}$ and "sleep hygiene" (controlling of all behavioral and environmental factors that precede sleep). ${ }^{12,13}$

In general, the reciprocal relationship between sleep and headache is well known in both adults and in the developmental age. ${ }^{6,14-24}$ Moreover, chronobiological aspects might interfere with the clinical phenotype in migraine, and a sort of desynchronization between the endogenous biological clock and lifestyle may impact the homeostatic function of sleep, promoting stress and accounting for the different levels of severity in migraine patients. This supports the hypothesis of an impairment of chronobiological rhythms occurring in those with migraine. ${ }^{25,26}$

In summary, there are different ways to approach the relationship between sleep and headache: (1) headache could be considered to be a result of sleep disorders; (2) headache may be the cause of the alteration of sleep; or (3) headache and sleep could be thought of as an expression of a common pathogenic process. ${ }^{27,28}$ Thus, the nature of sleep disorders associated with headaches may be considered as varied and manifold. In fact, children affected by migraine tend to show many sleep disorders, such as insufficient sleep duration, room-sharing or bed-sharing with parents (co-sleeping), increased latency to sleep onset, reluctance to go to sleep, decreased duration of night's sleep, nocturnal awakenings, anxiety regarding falling asleep, parasomnias, respiratory disorders, excessive daytime sleepiness (EDS) (not only during the attacks but also in the interictal period), and enuresis. . $^{5,8,29}$

Therefore, the aim of study is to assess the rate of parent reported sleep disorders and self-reported sleepiness in a large pediatric sample of individuals affected by migraine without aura (MoA).

\section{Materials and methods Study population}

Data were collected from a pilot group (10 with MoA and 10 control children) and used to perform sample size calculation. The desired power was set at 0.80 and the desired error was set at 0.05 . The sample size was calculated using online software (DSS Research, Fort Worth, TX, USA: http://www.dssresearch.com/toolkit/sscalc/size_a2.asp). The sample size required was found to be 23 subjects for each group, but there was an opportunity to recruit more patients in order to strengthen our findings. Therefore, the study population consisted of 271 children (122 females, 149 males) aged between 6 and 13 years (mean, $8.74 \pm 3.06$ years) consecutively referred for MoA at the Center for Childhood Headache of the Unit of Child and Adolescent Neuropsychiatry of the Second University of Naples, Naples,
Italy. The average frequency of the attacks was six per month (standard deviation [SD], 3.03). The diagnosis of MoA was made according to the pediatric criteria of the International Headache Society Classification (2004). ${ }^{30}$

Exclusion criteria were mental retardation, association of different types of headache, changes in neuroradiological (magnetic resonance imaging or computed tomography scan) and neurophysiological (wake and sleep electroencephalogram) aspects, concomitant neurological (eg, epilepsy, movement disorders, and cerebral palsy) or psychiatric (eg, schizophrenia, mood and anxiety disorders, psychosis, eating disorders, and attention deficit hyperactivity disorder) disorders, metabolic disease, obesity, and signs of sleep-related breathing disorders.

Following recruitment, there was a 4-month run-in period to verify the headache characteristics. For admission in this study, a length of headache occurrences over at least 8 months was required with a minimum of four attacks monthly, each lasting for a duration of 1 hour, according to International Classification of Headache Disorders (ICHD-II) criteria. ${ }^{30}$ All parents gave their written informed consent.

The results were compared with the findings obtained from a sample of 305 healthy controls (142 females, 163 males; mean age, $8.39 \pm 2.45$ years) recruited from schools in the Campania region, Italy. Subjects in both groups were recruited from the same urban area and were all of Caucasian origin and of middle socioeconomic status.

The Clinical Departmental University Ethics Committee of the Second University of Naples approved the study (protocol number 69/2012), which was conducted according to the criteria of the Declaration of Helsinki, as modified in 2000.

\section{Main outcome measures}

Sleep Disturbance Scale for Children

To evaluate the sleep habits and disturbances, all subjects' mothers filled out the Sleep Disturbance Scale for Children (SDSC), ${ }^{31}$ a standardized questionnaire for the assessment of sleep problems in the developmental age with a high internal consistency $(\alpha=0.79)$ and test/retest reliability $(r=0.71){ }^{31}$ it is considered effective at assessing sleep disturbances in a similar age group as the present study. ${ }^{32,33}$ The SDSC consists of 26 items grouped into six subscales: disorders in initiating and maintaining sleep, sleep breathing disorders, disorders of arousal, sleep-wake transition disorders, disorders of excessive somnolence, and nocturnal hyperhidrosis. This scale, both the original ${ }^{34,35}$ and modified versions, is widely used in school-aged children. ${ }^{36}$ According to the SDSC validation criteria, ${ }^{31}$ scores $\geq 71$ for SDSC total score, $\geq 17$ for disorders in initiating and maintaining sleep, $\geq 7$ for sleep breathing 
disorders, $\geq 6$ for disorders of arousal, $\geq 14$ for sleep-wake transition disorders, $\geq 13$ for disorders of excessive somnolence, and $\geq 7$ for nocturnal hyperhidrosis were considered as pathological.

\section{Pediatric Daytime Sleepiness Scale}

To check the degree of subjective perceived daytime sleepiness, all subjects were administered the Pediatric Daytime Sleepiness Scale (PDSS). The PDSS is an eight-item, selfreported Likert-type questionnaire that measures daytime sleepiness in school age populations, with possible scores ranging from $0-32$. Higher PDSS scores indicate greater daytime sleepiness. Along with being easy to administer, score, and interpret, the PDSS has a high internal consistency $(\alpha)$ of at least 0.80 in split-half samples and acceptable factor loadings $(>0.4) .{ }^{37}$ Furthermore, the duration of nocturnal sleep was evaluated through ad hoc questions.

\section{Statistical analysis}

In order to compare the characteristics (age, sex, and $\mathrm{z}-\mathrm{BMI}$ ) and the SDSC and PDSS results of two samples, the Chi-square test and $t$-test were applied where appropriate. $P$-values $<0.05$ were considered statistically significant. All data were coded and analyzed using the commercially available STATISTICA 6.0 package for Windows (StatSoft, Inc, Tulsa, OK, USA).

\section{Results}

The two study groups were matched for age (mean, $8.74 \pm 3.06$ vs $8.39 \pm 2.45 ; P=0.124)$, sex $(P=0.775)$, and z-BMI (mean, $0.53 \pm 0.14$ vs $0.56 \pm 0.28 ; P=0.107$ ) (Table 1$)$. Parents of children with migraine reported a higher rate of sleep disorders symptoms (SDSC TOT, 32.48\% of MoA vs $4.19 \%$ of controls; $P<0.001$ ), disorders of initiating and maintaining sleep $(37.11 \%$ of MoA vs $9.17 \%$ of controls; $P<0.001)$, and disorders of arousal $(59.32 \%$ of MoA vs $10.15 \%$ of controls; $P<0.001$ ) than parents of control sample. No significant differences were found in the disorders of excessive somnolence subscale (13.08\% of MoA vs $11.41 \%$ of controls) (Table 2).

Conversely, in the PDSS test, children with MoA had higher scores $(24.67 \pm 3.19$ vs $11.94 \pm 4.81 ; P<0.001)$ and a reduction in referred sleep mean duration $(469.83 \pm 98.112 \mathrm{vs}$ $527.94 \pm 83.02 ; P<0.001)$ than typically developing children (Table 3).

\section{Discussion}

In recent years, several studies have been devoted primarily to investigating the relationship between migraine and sleep
Table I The age, sex, and z-score body mass index (z-BMI) differences between the group of children affected by migraine without aura (MoA) and typically developing children (normal)

\begin{tabular}{llll}
\hline & $\begin{array}{l}\text { MoA } \\
(\mathbf{N}=\mathbf{2 7} \text { I) }\end{array}$ & $\begin{array}{l}\text { Normal } \\
(\mathbf{N}=\mathbf{3 0 5})\end{array}$ & $\mathbf{P}$ \\
\hline Age & $8.74 \pm 3.06$ & $8.39 \pm 2.45$ & 0.124 \\
Sex ratio (M/F) & $149 / 122$ & $163 / 142$ & 0.775 \\
z-BMI & $0.53 \pm 0.14$ & $0.56 \pm 0.28$ & 0.107 \\
(z-score body mass index) & & & \\
\hline
\end{tabular}

Notes: The t-test and Chi-square test, where appropriate, were applied. $P$-values $<0.05$ were considered statistically significant.

disorders, suggesting a significant correlation between these two conditions. ${ }^{6,22,38}$ This was also confirmed in this study by the higher prevalence, according to parents' reports, of sleep disorder symptoms (SDSC TOT, $P<0.001$ ) in the MoA group than in the control group.

Uncertain and not yet clarified mechanisms seem to be the biological basis for this association, although many studies tend to support a sort of cause-effect and/or, alternatively, a common pathogenesis ${ }^{39}$ as a putative effect of an alteration in the control of the core of the serotoninergic median raphe, which is important in circadian rhythm regulation and in genesis migraine. ${ }^{6}$

Herein, our findings regarding the higher prevalence of disorders in initiating and maintaining sleep $(P<0.001)$ and disorders of arousal $(P<0.001)$ corroborate the results of a recent polysomnographic study that suggested the presence of a peculiar macrostructural alteration in sleep organization in children with migraine, such as increased sleep latency, decreased total sleep time, and nocturnal awakenings rate, as well as the presence of respiratory disorders. ${ }^{6}$

Table 2 The differences between the group of children affected by migraine without aura (MoA) and typically developing children (normal) in pathological score (PS) on the Sleep Disturbance Scale for Children (SDSC)

\begin{tabular}{|c|c|c|c|c|}
\hline & $\begin{array}{l}\text { MoA } \\
(N=27 \text { I }) \\
(\%)\end{array}$ & $\begin{array}{l}\text { Normal } \\
(\mathrm{N}=305) \\
(\%)\end{array}$ & Chi-square & $P$ \\
\hline DIMS_PS & 37.11 & 9.17 & 65.537 & $<0.001$ \\
\hline SBD_PS & 4.12 & 2.98 & 0.247 & NS \\
\hline DA_PS & 59.32 & 10.15 & 154.393 & $<0.001$ \\
\hline SWTD_PS & 9.57 & 10.61 & 0.048 & NS \\
\hline DOES_PS & 13.08 & II.4I & $0.07 I$ & NS \\
\hline SHY_PS & 6.47 & 7.91 & 0.338 & NS \\
\hline SDSC TOTAL_PS & 32.48 & 4.19 & 77.035 & $<0.001$ \\
\hline
\end{tabular}

Notes: The Chi-square test was applied. P-values $<0.05$ were considered statistically significant.

Abbreviations: DA, disorders of arousal; DIMS, disorders in initiating and maintaining sleep; DOES, disorders of excessive somnolence; NS, not significant; $\mathrm{SBD}$, sleep breathing disorders; SHY, nocturnal hyperhidrosis. 
Table 3 The mean score of the Pediatric Daytime Sleepiness Scale (PDSS) and total sleep duration (expressed in minutes) of children affected by migraine without aura (MoA) and typically developing children (normal)

\begin{tabular}{lcll}
\hline & $\begin{array}{l}\text { MoA } \\
(\mathbf{N}=\mathbf{2 7})\end{array}$ & $\begin{array}{l}\text { Normal } \\
\mathbf{( N = 3 0 5 )} \\
(\%)\end{array}$ & $\mathbf{P}$ \\
\hline PDSS & $24.67 \pm 3.19$ & $\mathrm{II} .94 \pm 4.8 \mathrm{I}$ & $<0.00 \mathrm{I}$ \\
$\begin{array}{l}\text { Total sleep time } \\
\text { duration (min) }\end{array}$ & $469.83 \pm 98 . \mathrm{II} 2$ & $527.94 \pm 83.02$ & $<0.00 \mathrm{I}$ \\
\hline
\end{tabular}

Notes: The $t$-test was applied. $P$-values $<0.05$ were considered statistically significant.

On the other hand, our results may confirm the role of MoA in disorders of initiating and maintaining sleep and in excessive daytime sleepiness, as suggested in a previous study. ${ }^{5}$ These findings may suggest a putative role in the negative influences on academic performance, mood regulation, and behavior in the developmental age. ${ }^{40}$ For this reason, we have focused on MoA and not on other types of headaches because there are few specific studies about the relationship between MoA and sleep disturbances.

In 2010, Ødegård et al reported that subjects with chronic headache were 17 times more likely to have severe sleep disturbances, and the association was stronger for chronic migraine than for chronic tension type headache, ${ }^{41}$ pinpointing the impact of migraine pathology on body homeostasis. ${ }^{42,43}$ Migraine is a common condition in the developmental age with a prevalence between 3\% and $10.6 \%{ }^{21}$ in school-age children, and EDS can be considered to be a frequent and disabling symptom, ranging from $10 \%$ to $20 \%$ in the general population and greater in the youth and old age groups. ${ }^{44}$

Alternatively, EDS is a symptom with specific underlying pathogenic mechanisms, and it could be generally caused by many disturbances, including obesity and/or metabolic syndrome, ${ }^{5,45,46}$ mood disorders, sleep related breathing disorders ${ }^{47}$ and poor sleep efficiency. In this light, our results about the higher prevalence of sleep disorders in children with migraine can be interpreted as such disorders being a potential trigger of EDS in these subjects. Conversely, somnolence may be considered as part of the different phases of migraine attacks, even if there have been few studies on this. ${ }^{48-52}$

With regard to the presence of EDS subjectively perceived in the daily life activities of migraine patients, compared to previous studies that found a higher percentage of EDS in an adult population of migraineurs, ${ }^{48,49}$ our MoA children experienced EDS more frequently than healthy controls.
On the other hand, several brain structures and neurotransmitter systems involved in sleep regulation may be involved in the genesis of primary headaches like migraine, such as monoamine, acetylcholine, GABAergic structures, the orexin-hypocretin system, prostaglandins (viz PGD2), cytokines (IL1), and adenosine, ${ }^{51}$ but an alternative theory is the other way around, postulating that poor sleep can alter pain processing and therefore could alter the pain threshold. ${ }^{51}$

Conversely another interesting finding of the present study is the discrepancy between the questionnaire results (filled out by parents) and the PDSS about the presence of daytime somnolence. This may be explained by the fact that migraineurs tend to perceive subjectively a high grade of tendency for sleep not explained by other medical conditions. We can then hypothesize that this report can be interpreted as a sort of disequilibrium in excitatory and inhibitory neurotransmissions in children affected by migraine.

\section{Conclusion}

The following limitations of this study need to be taken into account: subjects were recruited from a specific region in Southern Italy, and the assessment of sleep disorders and excessive daytime sleepiness was not performed using objective methods such as polysomnography and/or the Multiple Latency Sleep Test. However, our study does identify differences in sleep habits and a high prevalence of daytime somnolence in children affected by MoA, suggesting the need for routine sleep screening in pediatric management of children with migraine.

\section{Disclosure}

The authors report no conflicts of interest in this work.

\section{References}

1. Edward Liveing. On Megrim, Sick-Headache and Some Allied Disorders: A Contribution to the Pathology of Nerve-Storms. London: J. and A. Churchill; 1873. Available from: http://www.archive.org/details/ onmegrimsickhea00livegoog. Accessed December 27, 2012.

2. Kernick D, Campbell J. Measuring the impact of headache in children: a critical review of the literature. Cephalalgia. 2008;29:3e16.

3. Esposito M, Pascotto A, Gallai B, et al. Can headache impair intellectual abilities in children? An observational study. Neuropsychiatr Dis Treat. 2012;8:509-513.

4. Esposito M, Verrotti A, Gimigliano F, et al. Motor coordination impairment and migraine in children: a new comorbidity? Eur J Pediatr. 2012;171(11):1599-1604.

5. Carotenuto M, Guidetti V, Ruju F, Galli F, Tagliente FR, Pascotto A. Headache disorders as risk factors for sleep disturbances in school aged children. J Headache Pain. 2005;6(4):268-270.

6. Vendrame M, Kaleyias J, Valencia I, Legido A, Kothare SV. Polysomnographic findings in children with headaches. Pediatr Neurol. 2008;39(1):6-11. 
7. Carotenuto M, Esposito M, Precenzano F, Castaldo L, Roccella M. Cosleeping in childhood migraine. Minerva Pediatr. 2011;63(2): 105-109.

8. Carotenuto M, Esposito M, Pascotto A. Migraine and enuresis in children: an unusual correlation? Med Hypotheses. 2010;75(1):120-122.

9. Verrotti A, Agostinelli S, D'Egidio C, et al. Impact of a weight loss program on migraine in obese adolescents. Eur J Neurol. 2013; 20(2):394-397.

10. Esposito M, Ruberto M, Pascotto A, Carotenuto M. Nutraceutical preparations in childhood migraine prophylaxis: effects on headache outcomes including disability and behaviour. Neurol Sci. 2012;33(6):1365-1368.

11. Esposito M, Carotenuto M. Ginkgolide B complex efficacy for brief prophylaxis of migraine in school-aged children: an open-label study. Neurol Sci. 2011;32(1):79-81.

12. Bruni O, Galli F, Guidetti V. Sleep hygiene and migraine in children and adolescents. Cephalalgia. 1999;19(Suppl 25):57-59.

13. Carotenuto M, Gallai B, Parisi L, Roccella M, Esposito M. Acupressure therapy for insomnia in adolescents: a polysomnographic study. Neuropsychiatr Dis Treat. 2013;9:157-162.

14. Aguggia M, Cavallini M, Divito N, et al. Sleep and primary headaches. Neurol Sci. 2011;32(Suppl 1):S51-S54.

15. Kristiansen HA, Kværner KJ, Akre H, Overland B, Russell MB. Migraine and sleep apnea in the general population. $J$ Headache Pain 2011;12(1):55-61.

16. Lucchesi LM, Speciali JG, Santos-Silva R, Taddei JA, Tufik S, Bittencourt LR. Nocturnal awakening with headache and its relationship with sleep disorders in a population-based sample of adult inhabitants of Sao Paulo City, Brazil. Cephalalgia. 2010;30(12):1477-1485.

17. Mitsikostas DD, Viskos A, Papadopoulos D. Sleep and headache: the clinical relationship. Headache. 2010;50(7):1233-1245.

18. Yeung WF, Chung KF, Wong CY. Relationship between insomnia and headache in community-based middle-aged Hong Kong Chinese women. J Headache Pain. 2010;11(3):187-195.

19. Lovati C, D’Amico D, Raimondi E, Mariani C, Bertora P. Sleep and headache: a bidirectional relationship. Expert Rev Neurother. 2010;10(1):105-117.

20. Brennan KC, Charles A. Sleep and headache. Semin Neurol. 2009; 29(4):406-418.

21. Bruni O, Russo PM, Ferri R, Novelli L, Galli F, Guidetti V. Relationships between headache and sleep in a non-clinical population of children and adolescents. Sleep Med. 2008;9(5):542-548.

22. Gilman DK, Palermo TM, Kabbouche MA, Hershey AD, Powers SW. Primary headache and sleep disturbances in adolescents. Headache. 2007;47(8):1189-1194.

23. Isik U, Ersu RH, Ay P, et al. Prevalence of headache and its association with sleep disorders in children. Pediatr Neurol. 2007;36(3): 146-151.

24. Rains JC, Poceta JS. Headache and sleep disorders: review and clinical implications for headache management. Headache. 2006; 46(9):1344-1363.

25. Gori S, Morelli N, Maestri M, et al. Sleep quality, chronotypes and preferential timing of attacks in migraine without aura. $J$ Headache Pain. 2005;6:258-2560.

26. Vyazovskiy VV, Tobler I. The temporal structure of behaviour and sleep homeostasis. PLoS One. 2012;7(12):e50677.

27. Pavia T, Batista A, Martins P, et al. The relationship between headaches and sleep disturbances. Headache. 1995;35:590-596.

28. Pavia T, Farinha A, Martins A, et al. Chronic headaches and sleep disorders. Arch Intern Med. 1997;157:1701-1705.

29. Lateef T, Swanson S, Cui L, Nelson K, Nakamura E, Merikangas K. Headaches and sleep problems among adults in the United States: findings from the National Co-morbidity Survey-Replication study. Cephalalgia. 2011;31(6):648-653.

30. Headache Classification Subcommittee of the International Headache Society. The International Classification of Headache Disorders, 2nd ed. Cephalalgia. 2004;24(Suppl 1):9-160.
31. Bruni O, Ottaviano S, Guidetti V, et al. The Sleep Disturbance Scale for Children (SDSC). Construction and validation of an instrument to evaluate sleep disturbances in childhood and adolescence. J Sleep Res. 1996;5:251-261.

32. Blunden SL, Chervin RD. Sleep problems are associated with poor outcomes in remedial teaching programmes: a preliminary study. J Paediatr Child Health. 2008;44:237-242.

33. Blunden S, Lushington K, Lorenzen B, Ooi T, Fung F, Kennedy D. Are sleep problems under-recognised in general practice? Arch Dis Child. 2004;89:708-712.

34. Bruni O, Ferini-Strambi L, Russo PM, et al. Sleep disturbances and teacher ratings of school achievement and temperament in children. Sleep Med. 2006;7:43-48.

35. Eitner S, Urschitz MS, Guenther A, et al. Sleep problems and daytime somnolence in a German population-based sample of snoring schoolaged children. J Sleep Res. 2007;16:96-101.

36. Spruyt K, Cluydts R, Verleye GB. Pediatric sleep disorders: exploratory modulation of their relationships. Sleep. 2004;27:495-501.

37. Drake C, Nickel C, Burduvali E, Roth T, Jefferson C, Pietro B. The Pediatric Daytime Sleepiness Scale (PDSS): sleep habits and school outcomes in middle-school children. Sleep. 2003;26:455-458.

38. Alberti A. Headache and sleep. Sleep Med Rev. 2006;10:431-437.

39. Rhee H, Miles MS, Halpern CT, et al. Prevalence and predictors of headaches in US adolescents. Pediatr Nurs. 2005;31:314-319, 350.

40. Bursztein C, Steinberg T, Sadeh A. Sleep, sleepiness, and behavior problems in children with headache. J Child Neurol. 2006;21:1012-1029.

41. Ødegård SS, Engstrøm M, Sand T, Stovner LJ, Zwart JA, Hagen K. Associations between sleep disturbance and primary headaches: the third Nord-Trøndelag Health Study. J Headache Pain. 2010;11(3): 197-206.

42. Sancisi E, Cevoli S, Vignatelli L, et al. Increased prevalence of sleep disorders in chronic headache: a case-control study. Headache. 2010;50(9):1464-1472.

43. Montagna P. Hypothalamus, sleep and headaches. Neurol Sci. 2006; 27(Suppl 2):S138-S143.

44. Young TB. Epidemiology of daytime sleepiness: definitions, symptomatology and prevalence. J Clin Psychiatry. 2004;65(S16):12-16.

45. Carotenuto M, Santoro N, Grandone A, et al. The insulin gene variable number of tandem repeats (INS VNTR) genotype and sleep disordered breathing in childhood obesity. J Endocrinol Invest. 2009;32(9):752-755.

46. Carotenuto M, Bruni O, Santoro N, Del Giudice EM, Perrone L, Pascotto A. Waist circumference predicts the occurrence of sleepdisordered breathing in obese children and adolescents: a questionnairebased study. Sleep Med. 2006;7(4):357-361.

47. Carotenuto M, Esposito M, Parisi L, et al. Depressive symptoms and childhood sleep apnea syndrome. Neuropsychiatr Dis Treat. 2012;8: 369-373.

48. Peres MF, Stiles MA, Siow HC, Silberstein SD. Excessive daytime sleepiness in migraine patients. J Neurol Neurosurg Psychiatry. 2005;76(10):1467-1468.

49. Barbanti P, Fabbrini G, Aurilia C, Vanacore N, Cruccu G. A casecontrol study on excessive daytime sleepiness in episodic migraine. Cephalalgia. 2007;27(10):1115-1159.

50. Stroe AF, Roth T, Jefferson C, et al. Comparative levels of excessive daytime sleepiness in common medical disorders. Sleep Med. 2010; 11(9):890-896.

51. Karthik N, Kulkarni GB, Taly AB, Rao S, Sinha S. Sleep disturbances in 'migraine without aura' - a questionnaire based study. J Neurol Sci. 2012;321(1-2):73-76.

52. Karthik N, Sinha S, Taly AB, Kulkarni GB, Ramachandraiah CT, Rao S. Alteration in polysomnographic profile in 'migraine without aura' compared to healthy controls. Sleep Med. Epub December 8, 2012. 


\section{Publish your work in this journal}

Neuropsychiatric Disease and Treatment is an international, peerreviewed journal of clinical therapeutics and pharmacology focusing on concise rapid reporting of clinical or pre-clinical studies on a range of neuropsychiatric and neurological disorders. This journal is indexed on PubMed Central, the 'PsycINFO' database and CAS.

The manuscript management system is completely online and includes a very quick and fair peer-review system, which is all easy to use. Visit http://www.dovepress.com/testimonials.php to read real quotes from published authors.

\footnotetext{
Submit your manuscript here: http://www.dovepress.com/neuropsychiatric-disease-and-treatment-journal
} 